\title{
Factors Associated with Healthcare Avoidance among US Adults: Analyses of the 2019 Healthy Information National Trends Survey
}

\author{
Bista $S^{1}$, Yu R$R^{2}$, Shete $S^{2,3,4 \# *}$ and Shastri SS ${ }^{1 * *}$ \\ ${ }^{1}$ Department of Health Disparities Research, University of \\ Texas MD Anderson Cancer Center, USA \\ ${ }^{2}$ Department of Biostatistics, University of Texas MD \\ Anderson Cancer Center, USA \\ ${ }^{3}$ Department of Epidemiology, University of Texas MD \\ Anderson Cancer Center, USA \\ ${ }^{4}$ Division of Cancer Prevention and Population Science, \\ University of Texas MD Anderson Cancer Center, USA \\ ${ }^{\#}$ Co-Senior Authors and Co-Communicating Authors \\ *Corresponding author: Surendra Shastri, \\ Department of Health Disparities Research, University \\ of Texas MD Anderson Cancer Center, 1400 Pressler St., \\ Houston, TX, 77030, USA
}

Sanjay Shete, 1400 Pressler Dr, Houston, TX 77030, USA

Received: October 20, 2021; Accepted: November 17, 2021; Published: November 24, 2021

\begin{abstract}
Background: Healthcare avoidance represents a significant impediment to health and well-being of individuals and society. Avoidance undermines positive healthcare-seeking and carries significant health and economic consequences including increased morbidity and mortality as well as loss of job or economic productivity.
\end{abstract}

Objective: The current study analyzed factors associated with healthcare avoidance.

Methods: Using the 2019 Healthy Information National Trends Survey, we conducted multiple logistic regression to identify factors associated with avoiding doctor's visit even when deemed necessary. Factors included sociodemographic, economic, access to health care, health care practices and trust to better understand medical care avoidance.

Results: Notably, $30.3 \%$ of Americans reported healthcare avoidance, and $29 \%$ reported "no/little/some trust" in medical information from the doctor. Greater likelihood of healthcare avoidance was observed among those who had less trust in medical information from their doctor, younger, and male. Significant health behavior predictors of healthcare avoidance included current smokers, those who had infrequent visits to health professionals, and those with better self-reported health.

Conclusions: By identifying multiple factors that may be associated with healthcare avoidance we can identify individuals at risk for avoidant behaviors and encourage positive health behavior.

Keywords: Healthcare avoidance; Trust in doctor; Health behaviors; Health promotion

\section{Introduction}

Health behaviors are intentional or unintentional actions taken by individuals that can shape and directly impact health and general well-being [1,2]. Adoption of positive, health-promoting behaviors such as healthy diet, regular physical activity, avoiding risky behaviors (e.g. smoking), and timely utilization of health-care services (e.g. regular check-ups, cancer screening) are associated with reduced risk of cancer, diabetes, cardiovascular disease and other health problems [3-6]. In contrast, unhealthy lifestyle choices, health risky behavior and underutilization of health-care services are known to have adverse health consequences [7]. Medical care-seeking behavior in particular is an action taken by individuals that address healthrelated symptoms including seeking help from a range of medical channels to prevent, allay and treat health issues [8]. Not seeking needed or necessary medical care or avoiding visits to healthcare facilities represents a significant impediment to health promotion and overall well-being [9]. Such behavior is encapsulated in the concept of healthcare avoidance, which remains a prevalent issue among US adult populations [9].

The term avoidance is defined as the act of turning away from a perceived threat that evokes emotional or physiological distress, and often discussed as a coping strategy during stressful situations [9]. In the healthcare context, avoidance can manifest as treatment refusal (e.g. canceling appointments) or nonadherence, denial of symptoms and/or diagnosis [9-11], or delayed treatment seeking [9,12]. Thus, treatment avoidance undermines positive healthcare-seeking behaviors and carries significant health and economic consequences $[10,13,14]$. Healthcare avoidance makes a critical contribution to late stage disease diagnosis and poor survival rate $[9,15,16]$. Avoidance in the form of delaying screening and treatment have been associated with increased likelihood of late-stage breast cancer diagnosis, acute symptoms for heart disease, mortality from HIV [10,15,17,18], longer duration and cost of hospitalization, and loss of work productivity $[17,19,20]$.

To better understand the phenomenon of healthcare avoidance, the Behavioral Model of Healthcare Service Use sheds insight into the social determinants of healthcare avoidance. Following this framework, healthcare avoidance is multifactorial, and a result of interplay of various individual and system-level factors [21]. Previous studies have related healthcare avoidance to sociodemographic factors 
(e.g. age, education, income), logistical barriers (e.g. lack of health insurance, scheduling issues), personal attitudes and beliefs (e.g. cancer worry, fatalistic attitude towards cancer) and provider-related factors (e.g. no usual source of care, provider distrust) [13,16,18,2224]. Studies examining predictors of treatment avoidance or delay have largely focused on patient populations such as those with breast cancer [10,25], rectal cancer [26], history of stroke [27], obesity [28], and diabetes and cardiovascular disease [29], or have narrowly focused on specific avoidant behaviors such as cancer screening and treatment $[9,30]$.

Although healthcare avoidance is a common occurrence, existing studies are limited in its scope to provide a comprehensive account of this dynamic and complex phenomenon [9]. There is a need to advance more studies that emphasize systematic identification of various characteristics or factors that influence healthcare avoidance. The current study examines correlates of healthcare avoidance among US adults using a large, nationally representative dataset. We include several predisposing factors including socio-demographic, economic, access to health care, health care practices and trust to broaden our understanding of the notion of medical care avoidance.

\section{Methods}

\section{Data and sample}

In this study, we analyzed publicly available data from National Cancer Institute's Health Information National Trends Survey 5 (HINTS 5) Cycle 3 (2019), which is a large-scale, household interview survey of noninstitutionalized US adults aged $\geq 18$ years. The purpose of HINTS was to investigate trends in the publics' attitudes, knowledge, and practices related to cancer-related healthcare access and use. Data collection was conducted from January 22 to April 30, 2019 , and we used available sampling weights to derive population estimates. Additional information about the survey development, design, and methodology are described elsewhere [31].

\section{Measures}

Dependent variable: We used a one item measure to assess healthcare avoidance (i.e., "some people avoid visiting their doctor even when they suspect they should. Would you say this is true for you, or not true for you?") That was rated as "True" or "Not True."

Independent variables: Demographic factors included age (i.e., 18-34 years, 35-49 years, 50-64 years, 65+), sex (male or female), race/ethnicity (non-Hispanic White, non-Hispanic Black, Hispanic, or non-Hispanic Other), and place of residence (rural or urban). Respondents that identified as non-Hispanic White, 65+ years, female, and living in urban areas were considered as the reference group for their respective categories.

Socioeconomic factors included annual household income $(<\$ 35,000, \$ 35,000-\$ 49,999, \quad \$ 50,000-\$ 74,999$, or $\geq \$ 75,000)$ and education ( $\leq$ high school graduate, post high school or some college graduate, and college or postgraduate). College or postgraduate and earning $<\$ 35,000 /$ year were the reference groups for their respective categories.

Lack of access to healthcare was measured with a single yes/ no question assessing health insurance status for a range of health insurance plans (i.e., "Are you currently covered by any of the following types of health insurance or health coverage plans?"). Listed insurance coverage included: "Insurance through a current or former employer or union", "Insurance purchased directly from an insurance company," "Medicare, for people 65 and older, people with certain disabilities," "Medicaid, Medical Assistance, or any kind of government-assistance plan for those with low incomes or a disability," "TRICARE or other military health care," "VA (including those who have ever used or enrolled for VA health care)," "Indian Health Service," and "Any other type of health insurance or health coverage plan." The "yes" response was used as reference.

Health-related practice was measured using two items related to frequency of healthcare visits and smoking status. Healthcare visit frequency was measured using a single frequency item (i.e., "In the past 12 months, not counting times you went to an emergency room, how many times did you go to a doctor, nurse, or other health professional to get care for yourself?") rated on a 5-point scale ("None," "1 or 2 times," "3 or 4 times," "5-9 times," $\geq 10$ times").

Smoking status was based on two questions related to cigarette smoking quantity (i.e., "Have you smoked at least 100 cigarettes in your entire life?") and frequency (i.e., "How often do you now smoke cigarettes?"). Participants were asked to rate quantity with a yes/ no, and frequency with a 3-point scale ("not at all," "some days," or "everyday"). Participants were placed into one of three categories: (1) never smoker ("no" response to quantity question), (2) former smoker ("yes" response to the quantity, and "not at all" response to frequency question), or (3) current smoker ("yes" response to the quantity, and "every day" or "some days" response to the frequency question).

Health status was based on a single item of self-reported general health (i.e., "In general, would you say your health is...") rated on a 5 -point scale ( 1 = excellent, 2 = very good, 3 = good, 4 = fair/poor $)$.

Trust in information from a doctor was assessed with a single item (i.e., "In general, how much would you trust information about health or medical topics from each of the following? A doctor") rated on a 4 -point scale ( $3=$ a lot, $2=$ some, $1=$ a little, or $0=$ not at all $)$. The "some," "a little," and "not at all" responses were combined.

\section{Statistical analyses}

Statistical analyses were conducted using survey weights to account for the HINTS data collection strategies. The computation of weights involved calculating household-level base weights; adjusting for non-response; calculating person-level weights; and calibrating the person-level weights to population counts using the American Community Survey (HINTS 2019). Weighted prevalence of healthcare avoidance was calculated by demographic, socioeconomic, health access, psychosocial, behavioral, and overall health characteristics. We conducted survey-weighted logistic regression to evaluate factors associated with healthcare avoidance. Statistical significance was declared when a 2-sided p-value was $\leq 0.05$. All analyses were conducted using SAS, version 9.4 and were weighted to be nationally representative.

\section{Results}

Table 1 summarizes weighted percentages of demographic, socioeconomic, health access, psychosocial, behavioral, and overall 
Table 1: Characteristics of survey population by healthcare avoidance.

\begin{tabular}{|c|c|c|c|c|c|}
\hline & \multirow{2}{*}{$\begin{array}{c}\text { Total } \\
\mathrm{N}\end{array}$} & \multicolumn{2}{|c|}{ Avoid } & \multicolumn{2}{|c|}{ Not Avoid } \\
\hline & & $n$ & wt \% & $n$ & wt \% \\
\hline Respondents & 5,247 & 1,303 & 30.5 & 3,944 & 69.5 \\
\hline \multicolumn{6}{|l|}{ Age groups (years) } \\
\hline $18-34$ & 668 & 243 & 41 & 425 & 59 \\
\hline $35-49$ & 948 & 317 & 33.7 & 631 & 66.3 \\
\hline $50-64$ & 1,626 & 400 & 29 & 1,226 & 71 \\
\hline$>=65$ & 1,903 & 317 & 16.6 & 1,586 & 83.4 \\
\hline \multicolumn{6}{|l|}{ Sex } \\
\hline Male & 2,212 & 598 & 35.4 & 1,614 & 64.6 \\
\hline Female & 3,035 & 705 & 25.8 & 2,330 & 74.2 \\
\hline \multicolumn{6}{|l|}{ Race/Ethnicity } \\
\hline Non-Hispanic white & 3,008 & 738 & 28.8 & 2,270 & 71.2 \\
\hline Non-Hispanic black & 663 & 126 & 29.3 & 537 & 70.7 \\
\hline Hispanic & 716 & 209 & 36.8 & 507 & 63.2 \\
\hline Non-Hispanic Asian & 221 & 58 & 31 & 163 & 69 \\
\hline Non-Hispanic Other & 162 & 58 & 47.3 & 104 & 52.7 \\
\hline \multicolumn{6}{|l|}{ Residence } \\
\hline Urban & 4,590 & 1,102 & 29.8 & 3,488 & 70.2 \\
\hline Rural & 657 & 201 & 34.6 & 456 & 65.4 \\
\hline \multicolumn{6}{|l|}{ Education } \\
\hline High school graduate or lower & 1,239 & 373 & 36.4 & 866 & 63.6 \\
\hline Post-high school or some college & 1,558 & 393 & 30.4 & 1,165 & 69.6 \\
\hline College graduate or postgraduate & 2,381 & 520 & 24.8 & 1,861 & 75.2 \\
\hline \multicolumn{6}{|l|}{ Income } \\
\hline$<35,000$ & 1,465 & 431 & 33.6 & 1,034 & 66.4 \\
\hline $35,000-49,999$ & 617 & 158 & 36.8 & 459 & 63.2 \\
\hline $50,000-74,999$ & 841 & 230 & 36.8 & 611 & 63.2 \\
\hline$\geq 75,000$ & 1,786 & 380 & 24.2 & 1,406 & 75.8 \\
\hline \multicolumn{6}{|l|}{ Smoking } \\
\hline Current & 603 & 199 & 40.5 & 404 & 59.5 \\
\hline Former & 1,384 & 295 & 29.5 & 1,089 & 70.5 \\
\hline Never & 3,216 & 795 & 28.9 & 2,421 & 71.1 \\
\hline \multicolumn{6}{|l|}{ Trust in Doctor } \\
\hline Some/A little/Not at all & 1,496 & 496 & 41.7 & 1,000 & 58.3 \\
\hline A lot & 3,622 & 778 & 25.7 & 2,844 & 74.3 \\
\hline \multicolumn{6}{|l|}{ Seeking Medical Care (past year) } \\
\hline 3 or 4 times & 1,552 & 323 & 21.7 & 1,229 & 78.3 \\
\hline 5 - 9 times & 832 & 126 & 20.1 & 706 & 79.9 \\
\hline 10 or more times & 515 & 90 & 27.4 & 425 & 72.6 \\
\hline \multicolumn{6}{|l|}{ General Health } \\
\hline Excellent & 618 & 107 & 22.5 & 511 & 77.5 \\
\hline Very good & 1,892 & 435 & 28 & 1,457 & 72 \\
\hline Good & 1,880 & 494 & 32.2 & 1,386 & 67.8 \\
\hline Fair/Poor & 709 & 262 & 39.4 & 569 & 60.6 \\
\hline \multicolumn{6}{|l|}{ Health insurance } \\
\hline Yes & 4,898 & 1,149 & 28.6 & 3,749 & 71.4 \\
\hline No & 271 & 134 & 52.9 & 137 & 47.1 \\
\hline
\end{tabular}

Note: Dependent variable. "Some people avoid visiting their doctor even when they suspect they should. Would you say this is true for you, or not true for you?". health characteristics of study population stratified by whether respondents agreed that they avoid visiting their doctor even when they suspect they should. Overall, 30.5\% endorsed healthcare avoidance with greater frequency among those between 18 and 34 years old (41\%), males (35.4\%), Hispanics (36.8\%), those living in rural areas (34.6\%), $\geq$ high school degree (36.4\%), annual household income $\$ 35,000-49,999$ (36.8\%) and \$50,000-75,999 (36.8\%), current smokers (40.5\%), those having some, little, or no trust in information coming doctors (41.7\%), those having visited a health professional $\geq 10$ in the past year (27.4\%), those reporting fair/poor health (39.4\%), and those without health insurance (52.9\%).

Results from a sample weighted logistic regression are presented in Table 2. A notable finding suggested that those having "some/ little/no" trust in information from doctors were more likely to avoid healthcare compared to those with "a lot" of trust ( $\mathrm{OR}=1.83$; $95 \% \mathrm{CI}=1.42-2.36$ ). Results also highlighted key demographic risk factors with those aged 18-34y compared to $\geq 65 \mathrm{y}$ were more than 3 times likely to avoid healthcare $(\mathrm{OR}=3.61 ; 95 \% \mathrm{CI}=2.46-5.30)$, and males were more likely than females to avoid healthcare $(\mathrm{OR}=$ $1.44 ; 95 \% \mathrm{CI}=1.11-1.86$ ). Significant self-reported health and health behavior results suggested that current smokers were more likely to avoid healthcare compared to never smokers $(\mathrm{OR}=1.47 ; 95 \% \mathrm{CI}=$ 1.04-2.09), and greater visits to a health professional was related to less avoidance. Specifically, those who reported 3-4 visits ( $\mathrm{OR}=0.53$; $95 \% \mathrm{CI}=0.35-0.76)$ and $5-9$ visits $(\mathrm{OR}=0.42 ; 95 \% \mathrm{CI}=0.23-0.75)$ were less likely to avoid healthcare compared to those reporting no visits in the past year. Lastly, compared to those with excellent health, self-reported fair/poor $(\mathrm{OR}=2.73 ; 95 \% \mathrm{CI}=1.63-4.57)$, good $(\mathrm{OR}=$ $1.97 ; 95 \% \mathrm{CI}=1.36-2.86)$, and very good health $(\mathrm{OR}=1.57 ; 95 \% \mathrm{CI}=$ 1.07-2.32) was related to greater healthcare avoidance.

\section{Discussion}

Avoiding necessary healthcare is a significant concern [9] that bears heavy public health and economic consequences. The primary aim of the current study was to identify key risk factors for healthcare avoidance when needed using nationally representative data. Notably, we found that almost 1 in 3 American adults (30.3\%) avoided healthcare even when they suspected they should. This highlighted a large group at-risk for worsening health.

A concerning finding from the study showed that $29 \%$ of the sample reported no/little/some trust in medical information from their doctor. While patients generally trust their doctor or healthcare provider as a critical source of health information compared to other communication channels [32,33], the high rates of medical mistrust point to a public health concern. Thus, the patient-doctor relationship and impressions of the doctor can have long lasting consequences. This is particularly important among higher risk groups given that those with poorer health and less education tend to initially seek medical advice from a doctor [32]. Individuals may also choose to avoid doctor visits to avoid seeking health related information [22]. There is empirical support for the inverse relationship between healthcare information seeking and avoidance $[9,22,30]$. Accordingly, trust in doctors as a viable source of medial information is an important determinant of whether one may choose to avoid healthcare when needed. 
Table 2: Associations between those who responded to "some people avoid visiting their doctor even when they suspect they should. Would you say this is true for you, or not true for you?" And characteristics of US adults.

\begin{tabular}{|c|c|c|c|}
\hline \multirow[b]{2}{*}{ Characteristics } & \multirow[b]{2}{*}{$\begin{array}{c}\text { Adjusted } \\
\text { OR }\end{array}$} & \multicolumn{2}{|c|}{$95 \% \mathrm{Cl}$} \\
\hline & & LL & UL \\
\hline \multicolumn{4}{|l|}{ Age group (years; Referent. >=65) } \\
\hline $18-34$ & $3.611^{*}$ & 2.461 & 5.299 \\
\hline $35-49$ & $2.36^{*}$ & 1.748 & 3.186 \\
\hline $50-64$ & $1.808^{\star}$ & 1.444 & 2.263 \\
\hline \multicolumn{4}{|l|}{ Sex (Referent. Female) } \\
\hline Male & $1.435^{*}$ & 1.105 & 1.864 \\
\hline \multicolumn{4}{|c|}{ Race/Ethnicity (Referent. Non-Hispanic white) } \\
\hline Hispanic & 1.035 & 0.735 & 1.457 \\
\hline Non-Hispanic Asian & 1.154 & 0.691 & 1.926 \\
\hline Non-Hispanic Other & 1.588 & 0.579 & 4.355 \\
\hline Non-Hispanic black & 0.725 & 0.47 & 1.117 \\
\hline \multicolumn{4}{|l|}{ Residence (Referent. Urban) } \\
\hline Rural & 1.193 & 0.851 & 1.672 \\
\hline \multicolumn{4}{|c|}{$\begin{array}{l}\text { Education (Referent. College graduate or } \\
\text { postgraduate) }\end{array}$} \\
\hline$<=$ High school graduate & 1.348 & 0.999 & 1.819 \\
\hline Post-high school or some college & 0.998 & 0.767 & 1.298 \\
\hline \multicolumn{4}{|l|}{ Income (Referent. $<\$ 35,000$ ) } \\
\hline$\$ 35,000-\$ 49,999$ & 1.361 & 0.853 & 2.172 \\
\hline$\$ 50,000-\$ 74,999$ & 1.282 & 0.909 & 1.806 \\
\hline$>=\$ 75,000$ & 0.775 & 0.545 & 1.103 \\
\hline \multicolumn{4}{|l|}{ Smoking (Referent. Never) } \\
\hline Current & $1.474^{\star}$ & 1.037 & 2.094 \\
\hline Former & 1.195 & 0.882 & 1.619 \\
\hline \multicolumn{4}{|c|}{$\begin{array}{l}\text { How much do you trust information about } \\
\text { health or medical topics from doctor? } \\
\text { (Referent. A lot) }\end{array}$} \\
\hline Some/A little/Not at all & $1.831^{\star}$ & 1.421 & 2.36 \\
\hline \multicolumn{4}{|c|}{$\begin{array}{l}\text { In the past } 12 \text { months, how many times did } \\
\text { you go to health professional to get care for } \\
\text { yourself? (Referent. None) }\end{array}$} \\
\hline 1 or 2 times & 1.109 & 0.777 & 1.582 \\
\hline 3 or 4 times & 0.526 & 0.352 & 0.787 \\
\hline 5 - 9 times & 0.417 & 0.231 & 0.753 \\
\hline 10 or more times & 0.583 & 0.308 & 1.104 \\
\hline \multicolumn{4}{|c|}{ General Health (Referent. Excellent) } \\
\hline Fair/Poor & $2.73^{\star}$ & 1.629 & 4.574 \\
\hline Good & $1.971^{*}$ & 1.36 & 2.856 \\
\hline Very good & $1.572^{\star}$ & 1.067 & 2.315 \\
\hline \multicolumn{4}{|l|}{ Health Insurance (Referent. Yes) } \\
\hline No & 1.658 & 0.98 & 2.805 \\
\hline
\end{tabular}

The result showing that medical mistrust was related to healthcare avoidance was consistent with previous research [34,23]. Specifically, we found that those who reported lower levels of trust in information about health or medical topics from a doctor were more likely to report avoiding visiting their doctor even when they suspect they should. Unsurprisingly, medical mistrust has been associated with increased avoidance and delayed care in studies of diverse groups in the United States [15,23]. Mollborn, Stepanova, and Cook (2005) found that most patients who have a trusting relationship with their regular care physician were less likely than those with less trust to report having unmet health care needs.

We found that specific health behaviors were related to healthcare avoidance. In particular, healthcare avoidance was higher among those who were current smokers and had poorer self-reported worse health status, and those who never visited a healthcare professional in the past 12 months. These findings are consistent with previous studies showing unhealthy behaviors (e.g., excessive alcohol consumption, smoking) are related to healthcare avoidance. This is concerning as this also places individuals at increased health risks $[35,36]$. Given that health behaviors cluster together (e.g., smokers are also more likely to report physically inactivity, poor sleep, and unhealthy diets [37], smoking may be indicative of poorer health and an array of health risk behaviors. For instance, one study that examined the association of smoking status with health-related quality of life showed that current smokers reported poorer health related quality of life than those who never smoked, and were more likely to drink heavily, to binge drink, and to report depressive and anxiety symptoms [37].

Prior studies have associated self-rated health with health service use $[38,39]$. In this study, respondents who rated their health status as poor/fair, compared to excellent, were almost 3 times more likely to report avoiding healthcare. This contrasts with previous research suggesting poorer physical and mental health status as a significant predictor of healthcare utilization [40], greater physician visits [39], and greater likelihood of receiving treatment [38].

Sex and age were the only sociodemographic characteristics that predicted healthcare avoidance. Specifically, males were more likely to report healthcare avoidance, which is consistent with findings that men tend to lead less healthy lifestyles and adopt fewer preventative and health-promoting beliefs and behaviors [41,42]. Men report fewer healthcare visits than women $[42,43]$ and are less likely to seek professional mental health services $[44,45]$. These differences may help to shed light on why men suffer from more severe chronic conditions, such as greater rates of cancer, heart disease, shorter lifespans $[41,42,44]$. Given that many of the health conditions for which men are at greater risk are preventable [42], public health efforts should seek to identify and confront the health-related beliefs, attitudes and practices that impede healthcare use that serves to place men at greater risk for poorer health outcomes.

An additional finding that younger adults were more likely to report avoiding healthcare is consistent with previous research $[15,22,40]$. Although younger adults are at lower risk for health problems compared to older adults, structural barriers to healthcare such as lack of health insurance may be a strong determinant of healthcare avoidance and delay in this group $[15,46]$. Because employmentbased insurance is the predominant form of healthcare access among individuals $<65 y$, healthcare use may be largely a function of occupation status and employment benefit [47]. Structurally, young adults are less likely to have health insurance coverage compared to 
older age groups given age cutoffs for subsidized health insurance $[46,47]$.

Although older individuals were less likely to avoid healthcare when needed, healthcare avoidance has particularly adverse health consequences for older adults [48] given more frequent chronic health problems and disability $[48,49]$. Unlike younger adults, healthcare cost and lack of health insurance coverage are not commonly identified reasons for healthcare avoidance [46]. Rather, healthcare avoidance among older adults is largely related to perceived health care quality and the relationship with healthcare providers (e.g., communication and rapport) [46]. This underscores the importance expanding our understanding of medical care avoidance to include psychosocial factors. For example, psychosocial factors may include emotional costs of healthcare (e.g. fear of cancer treatment, embarrassment disclosing symptoms to healthcare professionals, cultural taboo, and stigma), distrust of healthcare providers and/or medical science community, low self-efficacy and low perceived need to seek medical care $[13,23,24,29,34]$.

Although the current study did not find significant difference in healthcare avoidance by insurance status, healthcare cost remains a strong driving force for healthcare decisions.). The healthcare policy in the US does not guarantee universal coverage. The long-term economic and health consequences of avoiding medical healthcare on society and individuals is an important line of work that needs further exploration [9].

The study findings must be considered in light of the following limitations. First, the data were cross-sectional and based on participant self-report. As such, we cannot infer causality and the study measures were vulnerable to recall bias. Second, a one-item measure was used to assess healthcare avoidance. Future research should consider multidimensional measures that capture greater nuance. For example, it is important to consider how avoidance of preventive services (e.g. cancer screening) are distinct from avoidance of curative services [12]. Individuals seeking curative services are likely aware of and present with a host of symptoms [8] whereas individuals seeking preventive services may be non-symptomatic and healthy.

Third, quantitative data cannot holistically capture experiences of those avoiding healthcare. Future research should consider qualitative interviews to unpack the complex treatment seeking process and shed light on the context within which individuals engage in care-seeking behaviors. Although sociodemographic factors are widely cited antecedents of healthcare avoidance [9], individual characteristics such as racial/ethnic minority status, low income, and low education were not significantly associated with healthcare avoidance among the general population. Studies with different settings and populations have found differences in treatment seeking behavior by race/ethnicity and education level. One study found that Medicare beneficiaries with type 2 diabetes that reported lower levels of education (i.e. less than a high school diploma and high school degree) and of "other" racial/ethnic minority status (versus nonHispanic White) were more likely to avoid healthcare [50]. Continual investigation of avoidant behavior among those with specific health conditions and racial/ethnic will facilitate better understanding of those at risk for avoidance of needed medical care.
By elucidating factors associated with healthcare use avoidance we can promote healthcare usage among individuals more likely to avoid healthcare. To promote healthcare usage among those at-risk for missing or delaying doctor appointments, interventions should aim to increase knowledge and awareness of the benefits of timely health screenings and routine checkups. The Community Guide developed by the nonfederal Task Force on Community Preventive Services (Task Force) provides evidence-based recommendations to improve population health through the uptake of breast, cervical, and colorectal cancer screening [51]. For instance, the guide identified appointment reminders (e.g., in print, phone call) as an effective intervention to increase community awareness of cancer screening services, group or one-on-one community-based educational activities can effectively promote healthy lifestyle behaviors, and efforts to minimize economic and logistical barriers to cancer screening services such as lowering out of pocket costs or expansion of clinic visitation hours can promote screening behavior [52].

A promising intervention is to foster collaborative care between patient-provider dyads to encourage healthcare usage and increase patient engagement [13]. An important aspect of collaborative care is patient engagement and involvement in the management of their own health [53]. Interventions that adopt and implement a range of eHealth applications such as healthcare system web portals, mobile health communication, and telehealth medicine, that are interactive, user friendly and accessible to diverse consumers may enhance provider and patient access to critical health information, increase collaboration, and improve quality of care [54]. Moreover, the development of effective health communication interventions centered on patient involvement in their own health and safety can help foster trust and collaborative partnerships between patients and their providers [54].

\section{References}

1. Short SE and S Mollborn. Social Determinants and Health Behaviors: Conceptual Frames and Empirical Advances. Curr Opin Psychol. 2015; 5: 78-84.

2. Park $\mathrm{CL}$, et al. Positive and negative health behavior changes in cancer survivors: a stress and coping perspective. J Health Psychol. 2008; 13: 11981206.

3. Park CL, AE Gaffey. Relationships between psychosocial factors and health behavior change in cancer survivors: an integrative review. Ann Behav Med. 2007; 34: 115-134.

4. Lakka TA and C Bouchard. Physical activity, obesity and cardiovascular diseases. Handb Exp Pharmacol, 2005; 170: 137-163.

5. Blanchard $\mathrm{CM}$, et al. Association between current lifestyle behaviors and health-related quality of life in breast, colorectal and prostate cancer survivors. Psychology \& Health. 2004; 19: 1-13.

6. Michaud DS, et al. Physical activity, obesity, height, and the risk of pancreatic cancer. Jama. 2001; 286: 921-929.

7. Zwolinsky S, G Raine, S Robertson. Prevalence, Co-Occurrence and Clustering of Lifestyle Risk Factors among UK Men. Journal of Men's Health, 2016; 12: 15-24.

8. Oberoi S, et al. Understanding health seeking behavior. J Family Med Prim Care. 2016; 5: 463-464

9. Byrne SK. Healthcare avoidance: a critical review. Holist Nurs Pract. 2008; 22: $280-292$.

10. Reifenstein K. Care-seeking behaviors of African American women with breast cancer symptoms. Res Nurs Health. 2007; 30: 542-557. 
11. Moore PJ, et al. Psychosocial factors in medical and psychological treatment avoidance: the role of the doctor-patient relationship. J Health Psychol. 2004; 9: 421-433.

12. Khakbazan Z, et al. Help seeking behavior of women with self-discovered breast cancer symptoms: a meta-ethnographic synthesis of patient delay. PLoS One. 2014; 9: e110262.

13. Ye J, R Shim, G Rust. Health care avoidance among people with serious psychological distress: analyses of 2007 Health Information National Trends Survey. J Health Care Poor Underserved. 2012; 23: 1620-1629.

14. Asch S, et al. Why do symptomatic patients delay obtaining care for tuberculosis? Am J Respir Crit Care Med. 1998; 157: 1244-1248.

15. Spleen AM, et al. Health care avoidance among rural populations: results from a nationally representative survey. J Rural Health. 2014; 30: 79-88.

16. Kannan VD, PJ Veazie. Predictors of avoiding medical care and reasons for avoidance behavior. Med Care. 2014; 52: 336-345

17. Gallups SF, et al. Predictors of Adherence and Treatment Delays among African American Women Recommended to Receive Breast Cance Chemotherapy. Womens Health Issues. 2018; 28: 553-558.

18. Moser DK, et al. Reducing delay in seeking treatment by patients with acute coronary syndrome and stroke: a scientific statement from the American Heart Association Council on cardiovascular nursing and stroke council. Circulation. 2006; 114: 168-182.

19. Altice NF, EA Madigan. Factors associated with delayed care-seeking in hospitalized patients with heart failure. Heart Lung. 2012; 41: 244-254.

20. Kraft AD, et al. The health and cost impact of care delay and the experimenta impact of insurance on reducing delays. J Pediatr. 2009; 155: 281-285.e1.

21. Andersen RM. Revisiting the behavioral model and access to medical care: does it matter? J Health Soc Behav. 1995; 36: 1-10.

22. Persoskie A, RA Ferrer, WM Klein. Association of cancer worry and perceived risk with doctor avoidance: an analysis of information avoidance in a nationally representative US sample. J Behav Med. 2014; 37: 977-987.

23. Taber JM, B Leyva, A Persoskie. Why do people avoid medical care? A qualitative study using national data. J Gen Intern Med. 2015; 30: 290-297.

24. Vanderpool RC, B Huang. Cancer risk perceptions, beliefs, and physician avoidance in Appalachia: results from the 2008 HINTS Survey. J Health Commun. 2010; 15: 78-91.

25. Facione NC, et al. The self-reported likelihood of patient delay in breast cancer: new thoughts for early detection. Prev Med. 2002; 34: 397-407.

26. Ristvedt SL, KM Trinkaus. Psychological factors related to delay in consultation for cancer symptoms. Psychooncology. 2005; 14: 339-350.

27. Fuller R, N Dudley, J Blacktop. Avoidance hierarchies and preferences for anticoagulation--semi-qualitative analysis of older patients' views about stroke prevention and the use of warfarin. Age Ageing. 2004; 33: 608-611.

28. Drury CA, M Louis. Exploring the association between body weight, stigma of obesity, and health care avoidance. J Am Acad Nurse Pract. 2002; 14 $554-561$.

29. Larkey LK, et al. Hispanic cultural norms for health-seeking behaviors in the face of symptoms. Health Educ Behav. 2001; 28: 65-80.

30. Case DO, et al. avoiding versus seeking: the relationship of information seeking to avoidance, blunting, coping, dissonance, and related concepts. J Med Libr Assoc. 2005; 93: 353-362.

31. National Cancer Institute. Health Information National Trends Survey 5 (HINTS 5) Cycle 3 Methodology Report. 2019.

32. Volkman JE, et al. The National Cancer Institute's Health Information National Trends Survey [HINTS]: a national cross-sectional analysis of talking to you doctor and other healthcare providers for health information. BMC Fam Pract. 2014; 15: 111

33. Hesse BW, et al. Trust and sources of health information: the impact of the
Internet and its implications for health care providers: findings from the first Health Information National Trends Survey. Arch Intern Med. 2005; 165: 2618-2624.

34. Bynum SA, et al. Unwillingness to participate in colorectal cancer screening examining fears, attitudes, and medical mistrust in an ethnically diverse sample of adults 50 years and older. Am J Health Promot. 2012; 26: 295-300.

35. Henley SJ, et al. Vital Signs: Disparities in Tobacco-Related Cancer Incidence and Mortality - United States, 2004-2013. MMWR Morb Mortal Wkly Rep. 2016; 65: 1212-1218

36. Control CfD. Prevention, Targeting Tobacco Use: The Nation's Leading Cause of Death, 2005. US Department of Health and Human Services, ed. 2004.

37. Strine TW, et al. Health-related quality of life and health risk behaviors among smokers. Am J Prev Med. 2005; 28: 182-187.

38. Dhingra SS, et al. Determining prevalence and correlates of psychiatric treatment with Andersen's behavioral model of health services use. Psychiatr Serv, 2010; 61: 524-528.

39. Broyles RW, WJ McAuley, D Baird-Holmes. The medically vulnerable: their health risks, health status, and use of physician care. J Health Care Poor Underserved. 1999; 10: 186-200.

40. Babitsch B, D Gohl, T von Lengerke. Re-revisiting Andersen's Behaviora Model of Health Services Use: a systematic review of studies from 19982011. Psychosoc Med. 2012; 9: 11.

41. Hammond WP, et al. Masculinity, medical mistrust, and preventive health services delays among community-dwelling African-American men. J Gen Intern Med. 2010; 25: 1300-1308.

42. Courtenay WH. Constructions of masculinity and their influence on men's well-being: a theory of gender and health. Soc Sci Med. 2000; 50: 1385-1401.

43. Manuel JI. Racial/Ethnic and Gender Disparities in Health Care Use and Access. Health Serv Res. 2018; 53: 1407-1429.

44. Yousaf O, EA Grunfeld, MS Hunter. A systematic review of the factors associated with delays in medical and psychological help-seeking among men. Health Psychol Rev. 2015; 9: 264-276.

45. Galdas PM, F Cheater, P Marshall. Men and health help-seeking behaviour: literature review. J Adv Nurs, 2005; 49: 616-623.

46. Leyva B, JM Taber, AN Trivedi. Medical Care Avoidance Among Older Adults. J Appl Gerontol. 2020; 39: 74-85

47. Yabroff KR, et al. Financial Hardship Associated With Cancer in the United States: Findings From a Population-Based Sample of Adult Cancer Survivors. J Clin Oncol. 2016; 34: 259-267

48. Navaratnarajah A, SHD Jackson. The physiology of ageing. Medicine. 2017 45: 6-10.

49. Ward BW, JS Schiller. Prevalence of multiple chronic conditions among US adults: estimates from the National Health Interview Survey, 2010. Prev Chronic Dis. 2013; 10: E65.

50. $\mathrm{Ng} \mathrm{BP}$, et al. Factors Associated With Avoiding Health Care Among Community-Dwelling Medicare Beneficiaries With Type 2 Diabetes. Prev Chronic Dis. 2020; 17: E128.

51. Community Preventive Services Task Force. Cancer Screening: Client Reminders-Breast Cancer. 2010.

52. Task Force on Community Preventive Services, Recommendations for client- and provider-directed interventions to increase breast, cervical, and colorectal cancer screening. Am J Prev Med. 2008; 35: S21-S25.

53. Barello S, et al. eHealth for Patient Engagement: A Systematic Review. Frontiers in Psychology. 2016; 6.

54. Kreps GL, L Neuhauser. New directions in eHealth communication: opportunities and challenges. Patient Educ Couns. 2010; 78: 329-336. 